\title{
The evolution of molecular ruler analysis for characterizing telomere imbalances: from fluoresence in situ hybridization to array comparative genomic hybridization
}

Christa Lese Martin, PhD, Zafar Nawaz, PhD, Erin L. Baldwin, PhD, Elijah J. Wallace, MS, April N. Justice, MS, and David H. Ledbetter, PhD

\begin{abstract}
Submicroscopic telomere imbalances are a significant cause of mental retardation with or without other phenotypic abnormalities. We previously developed a set of unique telomere clones that identify imbalances in $3 \%$ to $5 \%$ of children with unexplained mental retardation and a normal karyotype. This targeted screening approach, however, does not provide information about the size or gene content of the imbalance. To enable such comprehensive characterization, a "molecular ruler" clone panel, extending up to $5 \mathrm{Mb}$ proximal to the first telomere clone for each chromosome arm, was developed. This panel of clones was successfully used to delineate the size of unbalanced telomere aberrations in a fluorescence in situ hybridization assay. However, the fluorescence in situ hybridization analysis was quite labor-intensive, and for many cases, the imbalance extended beyond our 5-Mb coverage. Therefore, to develop a more efficient and comprehensive method for characterizing telomere imbalances, we developed a custom oligonucleotide microarray consisting of high-density coverage of all telomere regions as well as a whole-genome backbone. Overall, 44 pathogenic imbalances studied by fluorescence in situ hybridization or oligonucleotide array showed a size range of $400 \mathrm{~kb}$ to $13.5 \mathrm{Mb}$. In four of these, the array detected additional interstitial imbalances adjacent to the telomere imbalance, demonstrating the usefulness of added probe coverage. In 10 cases with benign imbalances inherited from a normal parent, the size ranged from $170 \mathrm{~kb}$ to $1.6 \mathrm{Mb}$. These results demonstrate that array comparative genomic hybridization will aid in more efficient and precise characterization of telomere imbalances leading to the development of gene dosage maps at human telomere regions for genotype/phenotype correlations. Genet Med 2007:9(9):566-573.
\end{abstract}

Key Words: telomere imbalance, telomere, fluorescence in situ hybridization, array comparative genomic hybridization, molecular rulers

The telomeric regions of human chromosomes are frequently involved in chromosomal rearrangements due to their location at the terminus of each chromosome. Several of the first cytogenetically visible deletion syndromes described, including Wolf-Hirschhorn, cri du chat, and Miller-Dieker syndromes, were found to involve various telomeric regions. Based on these observations, it was hypothesized that smaller imbalances of these gene rich regions, which would be difficult to impossible to detect by routine G-banding analysis, could also contribute significantly to abnormal clinical pathology. ${ }^{1} \mathrm{~A}$

From the Department of Human Genetics, Emory University School of Medicine, Atlanta, Georgia.

Christa Lese Martin, PhD, Emory University, Department of Human Genetics, 615 Michael Street, Suite 301, Atlanta, GA 30322; E-mail: clmartin@genetics.emory.edu

Disclosure: The authors report no conflict of interest.

Submitted for publication May 8, 2007.

Accepted for publication June 27, 2007.

DOI: 10.1097/GIM.0b013e318149e1fc unique telomere clone set that could be used in molecular cytogenetic assays was envisioned for use in identifying these cryptic, or submicroscopic, imbalances.

As part of an international collaboration, a bacterial artificial chromosome (BAC) and/or P1-derived artificial chromosome (PAC) clone corresponding to each human telomere was identified, located just proximal to the subtelomeric repeat region. ${ }^{2,3}$ Each clone, therefore, represented the most distal unique DNA for each chromosome arm. Using this clone set, Knight et al. ${ }^{4}$ carried out the first large-scale study of 466 individuals with unexplained mental retardation and showed that $7.4 \%$ of individuals with moderate to severe mental retardation $(n=284)$ had a telomere imbalance compared with $0.5 \%$ of individuals with mild mental retardation $(n=182)$. Recently, our group reported the largest study to date to examine the frequency of telomere imbalances and demonstrate the clinical utility of this testing. ${ }^{5}$ Telomere fluorescence in situ hybridization (FISH) analysis of 11,688 patients with a developmental disability and a normal karyotype revealed the de- 
tection rate for clinically significant telomere abnormalities to be $2.5 \%$, with an additional $0.5 \%$ detection of apparently benign familial variants.

Telomere FISH analysis, although extremely valuable for clinical diagnostics, proved to be very labor-intensive for routine laboratory testing. Therefore, alternative methods were investigated, including array comparative genomic hybridization (array CGH). Several groups have now reported the successful transition of telomere analysis from FISH to array CGH with similar yields for deletions, but improved detection rates for small duplications. ${ }^{6-10}$ However, most of these initial arrays only contained limited clone coverage for each telomere region.

Although a targeted screening approach is useful for the initial detection of a telomere imbalance, it does not provide any information about the size or gene content of the imbalance, which makes the determination of clinical significance and comparison with other published cases difficult. Therefore, to create genotype/phenotype correlations for each human telomere region, we developed a set of "molecular ruler" clones for each telomere region to not only measure the size of the imbalance, but to also aid in examining the gene content.

Our initial molecular ruler clone panel included a BAC/PAC contig for the most distal $1 \mathrm{Mb}$ of each chromosome arm (starting at the unique telomere clone and moving proximal) and then a clone every $500 \mathrm{~kb}$ for the most distal $5 \mathrm{Mb} .{ }^{11}$ These clones were used in pilot FISH studies to interrogate imbalances involving 1p, 6q, 10q, 16p, 17p, and 22q..$^{8,11-13}$ Although these pilot studies only included a small number of cases per telomere, they demonstrated the utility of this high-density mapping for more accurate clinical interpretation of telomere imbalances. By determining the size of each telomere imbalance, pathogenic imbalances could be differentiated from those that were apparently benign variants, such as small deletions and duplications of $4 \mathrm{q}$ and $10 \mathrm{q} \cdot{ }^{8,11}$

In this study, we have extended the use of FISH with our molecular ruler clone set to characterize a larger set of unbalanced telomere rearrangements. However, these analyses, as well as those of other investigators, ${ }^{14,15}$ quickly led to the realization that many submicroscopic telomere imbalances were larger than $5 \mathrm{Mb}$ and that additional bioinformatics and clone validation work to identify sequential clones for FISH mapping was inefficient and time-consuming. Therefore, we used the mapping data from our well-characterized molecular ruler clone set to transition to array CGH using a custom designed oligonucleotide microarray with high-resolution coverage of each telomere region. Overall, 54 cases with 71 telomere imbalances were evaluated using either FISH or oligonucleotide array analysis.

\section{MATERIALS AND METHODS}

\section{Patient samples}

Individuals with developmental delay/mental retardation, a normal G-banded karyotype and an unbalanced telomere rearrangement, identified by telomere FISH or targeted microar- ray analysis, were recruited from clinical diagnostic laboratories and genetics clinics for participation in this study. Informed consent was obtained from all patients, and samples were collected following approved human subjects protocols through the Institutional Review Boards at the University of Chicago and Emory University.

\section{Genomic clone selection and FISH analysis}

The previously characterized set of unique telomere clones, which corresponds to the most distal unique DNA for each chromosome arm, was used as the starting point for developing the molecular rulers for each telomere region. ${ }^{3}$ The $\mathrm{BAC}$ and $\mathrm{PAC}$ clones included in the molecular ruler assay were selected based on published physical maps and publicly available genome resources, such as the University of California Santa Cruz Human Genome Browser (http://genome.ucsc.edu) ${ }^{16,17}$ and the integrated STS/radiation hybrid maps (NCBI Map Viewer, http://www.ncbi.nlm.nih.gov). ${ }^{18}$ For the most distal $1 \mathrm{Mb}$, a complete contig of overlapping clones covering the region was developed to analyze smaller imbalances. Genomic clones were then selected at $500-\mathrm{kb}$ intervals to cover the most distal $5 \mathrm{Mb}$ of each chromosome of interest. FISH was used to verify a subset of clones for cytogenetic position and unique localization (i.e., no crosshybridization signals to other chromosomes).

Bacterial stabs were obtained from the BACPAC Resource Center at Children's Hospital Oakland Research Institute (Oakland, CA) or from Invitrogen (Carlsbad, CA) and streaked onto LB plates with the appropriate antibiotic. DNA from each genomic clone was isolated from overnight cultures using an automated DNA isolation system (AutoGen 740, Integrated Separation Systems, Natick, MA) and directly labeled with either Spectrum Orange-dUTP or Spectrum GreendUTP (Abbott Molecular, Inc., Des Plaines, IL) using a standard nick translation reaction. Slide preparation, probe preparation, hybridization, and washing were carried out following previously described methods. ${ }^{11}$

\section{Array CGH analysis}

Two different oligonucleotide arrays were used for these experiments: a commercially available array (Agilent Human Genome CGH Microarray Kit 44B, Agilent Technologies, Santa Clara, CA) and a custom designed $4 \times 44 \mathrm{~K}$ oligonucleotide array (Agilent Technologies, manuscript in preparation). The oligonucleotides contained on both arrays were 60 mers. Based on the genomic location of the first unique FISH clone for each telomere, we targeted coverage on the custom array and included at least 10 oligonucleotides to cover the most distal unique telomere clone, providing a resolution of $50 \mathrm{~kb}$ for these regions. An oligonucleotide was then placed every $75 \mathrm{~kb}$ across the length of each chromosome arm starting adjacent to the telomere clone coverage. For these regions, when mapping known telomere rearrangements, we set the minimum number of oligonucleotides included in a region of loss or gain at three oligonucleotides, which allows us to achieve a resolution of 200 to $300 \mathrm{~kb}$. 
Array CGH analysis was carried out following the manufacturer's protocol (Agilent Technologies). Briefly, genomic DNA $(0.5-1.5 \mu \mathrm{g})$ was digested with AluI and RsaI (Promega Corporation, Madison, WI) for 2 hours. The DNA was labeled for 2 hours using random primers, Cy-3 and Cy-5-dUTP dyes, and Exo-Klenow fragment (Agilent Technologies). Patient DNA (labeled with Cy-3) was combined with a normal control DNA sample (labeled with Cy-5) of the opposite sex (Promega Corporation) and hybridized to the array in the presence of Cot-1 (Invitrogen). After a 24 -hour hybridization at $65^{\circ} \mathrm{C}$, the slides were washed and scanned using the GenePix Autoloader 4200AL (Molecular Devices, Sunnyvale, CA). Confirmatory FISH analyses of the breakpoints identified by oligonucleotide array were carried out using BAC clones from the molecular ruler panel, as described above.

BlueFuse microarray analysis software (BlueGnome Limited, Cambridge, UK) was used to examine the oligonucleotide array data. Normalization of the data was performed using Block Lowess. Regions of copy number alterations were detected using set thresholds for the channel ratios based on 2 or 3 SDs from the median of the autosomes. Channel 1 (Ch1) represents the patient sample and channel $2(\mathrm{Ch} 2)$ represents the normal control DNA. The thresholds for the $\log _{2}$ ratios were set at 0.26 for gains and -0.32 for deletions.

\section{RESULTS}

In this study, FISH and array CGH were employed to investigate telomere imbalances in 54 individuals with developmental delay/mental retardation who previously had a normal Gbanded karyotype. Because some of these rearrangements were derivative chromosomes involving more than one telomere region, the total number of unbalanced segments characterized was 71 . Although the majority of individuals examined carry a telomere imbalance that is pathogenic and related to their abnormal phenotypic findings, several cases were also examined where the imbalance was inherited from a phenotypically normal parent and are thus considered benign variants. Telomere mapping results are presented below in the context of these two categories.

\section{Pathogenic telomere imbalances}

Forty-four individuals with developmental delay/mental retardation were studied who carry a pathogenic telomere imbalance. Twenty-four cases had a pure de novo terminal deletion or duplication. As shown in Table 1, 23 de novo deletions, involving 14 different telomere regions, showed monosomy that ranged from $400 \mathrm{~kb}$ to $13.5 \mathrm{Mb}$. Figure 1, A shows oligonucleotide array analysis for Case 11 with a $5.9 \mathrm{Mb}$ deletion of the $8 \mathrm{p}$ telomere. Only one pathogenic de novo duplication was analyzed in this study, which was shown to be $2.8 \mathrm{Mb}$ in size (Case 24). Such small duplications are difficult to detect by FISH analysis. However, array analysis can accurately detect even small gains in copy number ( $\geq 100 \mathrm{~kb}$ ).

Sixteen cases involved an unbalanced derivative chromosome resulting from a rearrangement involving more than one
Table 1

Pathogenic telomere imbalances sized by FISH and oligonucleotide aCGH

\begin{tabular}{ccccc}
\hline & \multicolumn{2}{c}{ Size } & \\
\cline { 3 - 4 } Case no. & Telomere & $\begin{array}{c}\text { Deletion } \\
(\mathrm{Mb})\end{array}$ & $\begin{array}{c}\text { Duplication } \\
(\mathrm{Mb})\end{array}$ & $\begin{array}{c}\text { Mapped } \\
\text { by }\end{array}$ \\
\hline
\end{tabular}

Deletions

(de novo)

1
2
3
4
5
6
7
$8^{a}$
$9^{b}$

$1 \mathrm{p}$

$1 \mathrm{p}$

$1 \mathrm{q}$

$2 \mathrm{q}$

$3 q$

$4 \mathrm{p}$

$6 q$

$6 \mathrm{q}$

$6 \mathrm{q}$

10

11

12

13

14

15

16

17

18

19

20

$21^{c}$

22

23

Duplications

(de novo)

24

Derivatives

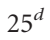

26

27

28

29

$30^{e}$

$31^{f}$

$32^{g}$

$33^{h}$

$34^{i}$

35

36

$4 q$

$25^{d}$

4.0
$6.5-7.0$
7.9
1.9
1.5
5.6
12.9
$400 \mathrm{~kb}$
$7.5-8.0$
10.5
5.9
10.0
1.5
1.4
1.1
1.6
4.5
4.0
$1.1-1.7$
1.8
1.2
1.1
1.6

FISH

FISH

aCGH

aCGH

aCGH

aCGH

aCGH

aCGH

FISH

aCGH

aCGH

aCGH

FISH

aCGH

aCGH

aCGH

aCGH

aCGH

FISH

aCGH

aCGH

FISH

aCGH

2.8

aCGH

$1 \mathrm{q} ; 6 \mathrm{q}$
$2 \mathrm{q} ; 22 \mathrm{q}$
$3 \mathrm{p} ; 9 \mathrm{p}$
$5 \mathrm{q} ; 10 \mathrm{q}$
$6 \mathrm{p} ; 6 \mathrm{q}$
$6 \mathrm{q} ; 21 \mathrm{q}$
$7 \mathrm{p} ; 16 \mathrm{p}$
$10 \mathrm{p} ; 10 \mathrm{q}$
$10 \mathrm{q} ; 16 \mathrm{p}$
$10 \mathrm{q} ; 16 \mathrm{p}$
$10 \mathrm{q} ; 17 \mathrm{q}$
$12 \mathrm{p} ; 17 \mathrm{p}$

$$
3.5 \text { (6q) }
$$$$
4.5 \text { (1q) }
$$

aCGH

2.4 (2q)

8.2 (22q)

aCGH

3.5 (9p)

$4.5(3 p)$

aCGH

2.7 (10q)

$6.8(5 q)$

aCGH

13.5 (6p)

10.0 (6q)

aCGH

7.1 (6q)

5.3 (21q)

aCGH

None (7p)

4.5 (16p)

FISH

8.5 (10q)

10.4 (10p)

aCGH

None (10q)

10.0 (16p)

FISH

None (10q)

10.0 (16p)

FISH

1.4 (10q)

4.5 (17q)

aCGH

1.35 (12p)

5.5 (17p)

aCGH

(Continued) 
Table 1

Continued

\begin{tabular}{ccccc}
\hline & & \multicolumn{3}{c}{ Size } \\
\cline { 3 - 4 } Case no. & Telomere & $\begin{array}{c}\text { Deletion } \\
(\mathrm{Mb})\end{array}$ & $\begin{array}{c}\text { Duplication } \\
(\mathrm{Mb})\end{array}$ & $\begin{array}{c}\text { Mapped } \\
\text { by }\end{array}$ \\
\hline $37^{j}$ & $14 \mathrm{p} ; 16 \mathrm{p}$ & None $(14 \mathrm{p})$ & $10.0(16 \mathrm{p})$ & FISH \\
$38^{k}$ & $16 \mathrm{p} ; 17 \mathrm{p}$ & $4-5(17 \mathrm{p})$ & $3.5(16 \mathrm{p})$ & FISH \\
39 & $\mathrm{Xp} ; 16 \mathrm{p}$ & $2.5(\mathrm{Xp})$ & $2.9(16 \mathrm{p})$ & aCGH \\
40 & $\mathrm{Xp} ; \mathrm{Xq}$ & $4.6(\mathrm{Xp})$ & $3.6(\mathrm{Xq})$ & aCGH \\
Complex & & & & \\
41 & $2 \mathrm{q}$ & 3.0 & 4.8 & aCGH \\
42 & $6 \mathrm{q}$ & 1.0 & $102 \mathrm{~kb}$ & aCGH \\
43 & $22 \mathrm{q}$ & $320 \mathrm{~kb}$ & 5.2 & aCGH \\
44 & $9 \mathrm{p} ; 20 \mathrm{p}$ & $10.4(9 \mathrm{p})$ & $1.2(9 \mathrm{p})$ & aCGH \\
& & & $4.3(20 \mathrm{p})$ & \\
\hline
\end{tabular}

FISH, fluorescence in situ hybridization; aCGH, array comparative genomic hybridization.

${ }^{a}$ Case $1 .{ }^{13}$

${ }^{b}$ Case $2 .{ }^{13}$

${ }^{c}$ Archer et al. ${ }^{19}$

${ }^{d}$ Case $3 .{ }^{13}$

${ }^{e}$ Cases 4 and $5 .{ }^{13}$

${ }^{f}$ Case $2 .{ }^{11}$

${ }^{8}$ Case $3 .{ }^{11}$

${ }^{h}$ Case $4 .{ }^{11}$

${ }^{i}$ Case $5 .{ }^{11}$

${ }^{j}$ Case $5 .{ }^{11}$

${ }^{k}$ Case $1 .{ }^{11}$

chromosomal segment. Although the majority of these were unbalanced translocations that were inherited from a normal parent who carried the balanced form of the translocation, a few cases were de novo. In this category, because more than one chromosomal region was involved in the rearrangement, 28 imbalances were characterized. For four cases, however, although two chromosomes were involved, only one telomere region had an abnormal copy number of unique sequence DNA, i.e., the derivative chromosome contained only a duplication of a telomere region translocated distal to an intact telomere. Figure 1, C shows an example of array results from an unbalanced telomere translocation between the long arms of chromosomes 5 and 10 (Case 28) resulting in trisomy for a $6.8 \mathrm{Mb}$ region of $5 \mathrm{q}$ and monosomy for a $2.7-\mathrm{Mb}$ region of $10 \mathrm{q}$.

The size of the monosomic segments associated with the derivative chromosomes ranged from 1.35 to $10 \mathrm{Mb}$. For the trisomic segments, sizes ranged from 2.9 to $10.4 \mathrm{Mb}$. As shown in Table 1, for many of the derivative chromosomes that were characterized, the size of the deleted segment was similar to the size of the duplicated segment. This observation explains why many of these large imbalances are still cryptic by G-banding because one region is deleted and replaced with another region of similar size and G-banding pattern.

Complex chromosome rearrangements, also listed in Table 1 , were discovered in four cases that were originally referred to our study as pure telomere deletions (Cases 41,42 , and 43 ) or an unbalanced telomere translocation (Case 44). In each of these cases, molecular ruler mapping by array CGH revealed an additional imbalance that was not detected by FISH analysis with a single telomere clone. Each case contained an additional interstitial duplication that was located adjacent to the originally identified deleted region. For example, Case 43 was referred to our study because of a 22q telomere deletion identified by FISH analysis. As shown in Figure 2, array analysis revealed a $5 \mathrm{Mb}$ duplication of the $22 \mathrm{q}$ telomere region in addition to the $320 \mathrm{~kb}$ deletion in this individual. By telomere FISH analysis, Case 44 was shown to have an unbalanced telomere translocation between the short arms of chromosomes 9 and 20 . Array analysis determined the size of the $9 p$ deletion to be $10.4 \mathrm{Mb}$ and the size of the $20 \mathrm{p}$ duplication to be $4.3 \mathrm{Mb}$, but also identified a 1.2-Mb duplication of 9p just proximal to the deleted region.

\section{Inherited benign variants}

Ten individuals with developmental delay/mental retardation examined in this study carried deletions or duplications that were inherited from a phenotypically normal parent. These cases are analogous to previous reports where the imbalance is assumed to be a benign variant because it is inherited from a normal parent. ${ }^{20-23}$ In fact, five of 10 benign telomere variants analyzed in this study have been previously reported, including deletions of $4 \mathrm{q}, 10 \mathrm{q}$, and $\mathrm{Yq}$ and duplications of 4q. ${ }^{5}$ By the same logic, the additional inherited imbalances described in this report, including deletions of $9 p$ and $21 q$ and duplications of $3 p$ and $15 q$, are most likely benign variants.

As shown in Table 2, six cases, involving five different chromosomes, were pure terminal deletions with sizes ranging from $300 \mathrm{~kb}$ to $1.6 \mathrm{Mb}$. Three cases were pure terminal duplications with sizes ranging from $170 \mathrm{~kb}$ to $1.5 \mathrm{Mb}$. One benign variant was caused by an unbalanced translocation (Case 54) resulting in a derivative 12 with $538 \mathrm{~kb}$ of the telomeric region of $15 q$ on the short arm of 12 with no associated loss of the $12 p$ telomere region. Figure 1, B shows array results from Case 54, which accurately detected and sized this small duplication.

Figure 1, D shows array results for chromosome 4 from Case 45 with a $1 \mathrm{Mb}$ benign $4 \mathrm{q}$ deletion using two different oligonucleotide array platforms, our custom designed oligonucleotide array and an off-the-shelf oligonucleotide array. Only our custom array, with enhanced coverage of the telomere regions, detected this $1-\mathrm{Mb}$ deletion. The off-the-shelf commercial array failed to detect this imbalance due to poor genomic coverage at this telomere region.

\section{Pathogenic versus benign telomere imbalances}

Although our molecular ruler clone set was originally designed with tiling path coverage of the most distal $1 \mathrm{Mb}$ because we hypothesized that most telomere imbalances would be small, only three of $61(5 \%)$ of the pathogenic imbalances that were delineated were $<1 \mathrm{Mb}$ in size. Thirty-nine of 61 (64\%) were $<5 \mathrm{Mb}$ in size. However, surprisingly, 22 of 61 (36\%) of the pathogenic imbalances were $>5 \mathrm{Mb}$, which extended beyond the initial upper limit for our molecular ruler coverage. In fact, the largest pathogenic imbalance was a $13.5 \mathrm{Mb}$ 

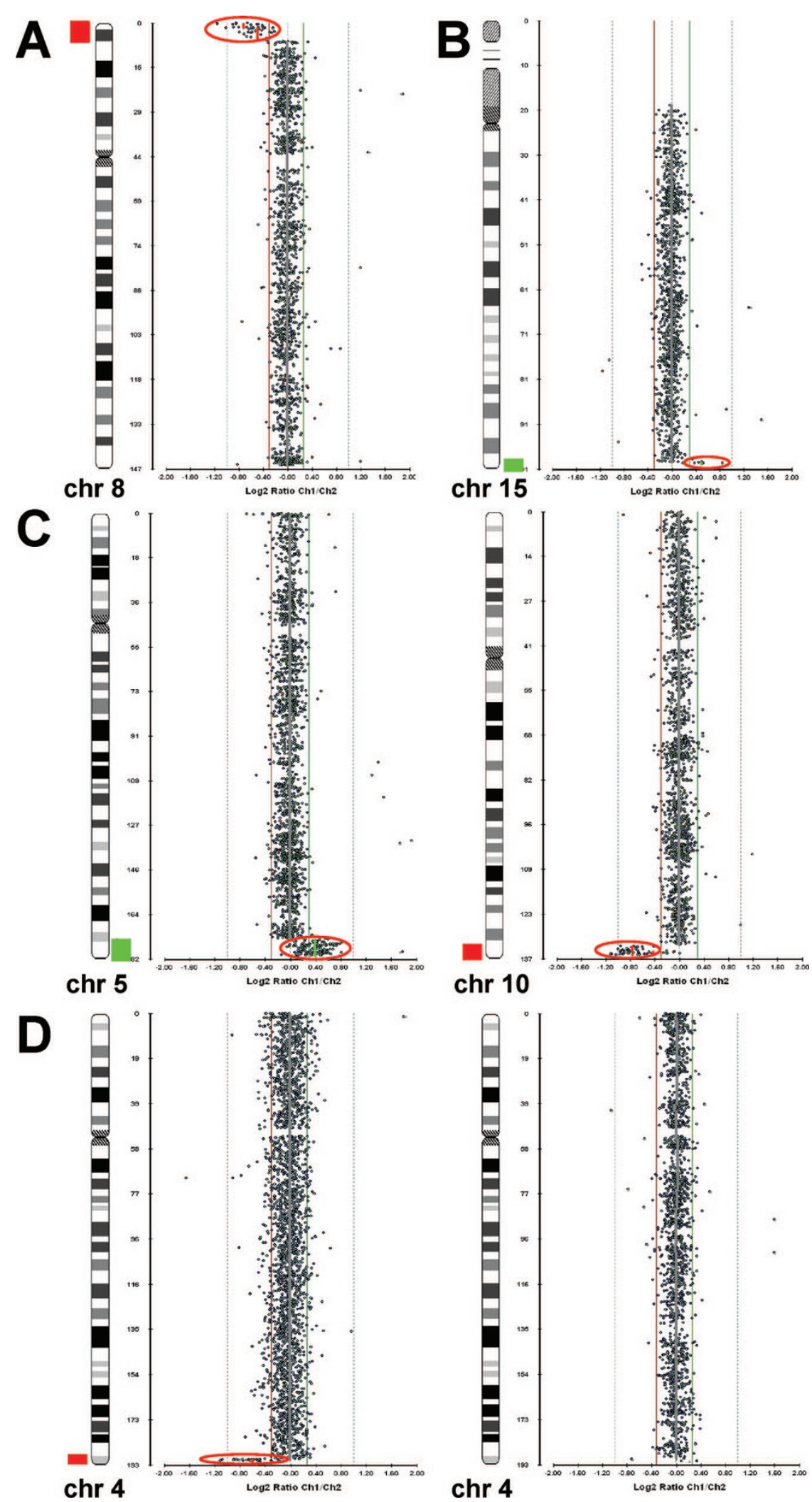

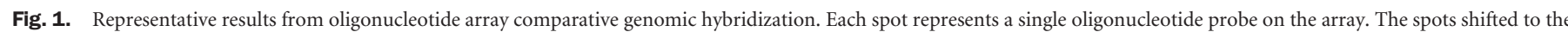

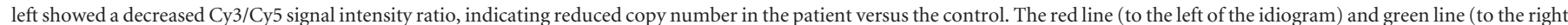

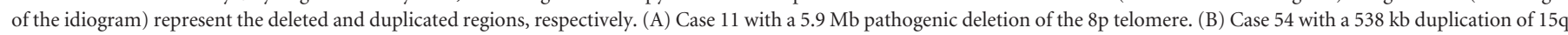

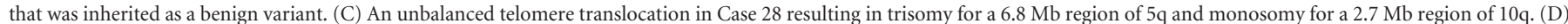

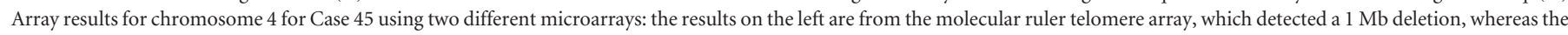
results on the right show results from an off-the-shelf commercial array that failed to detect the deletion due to incomplete telomeric coverage. 

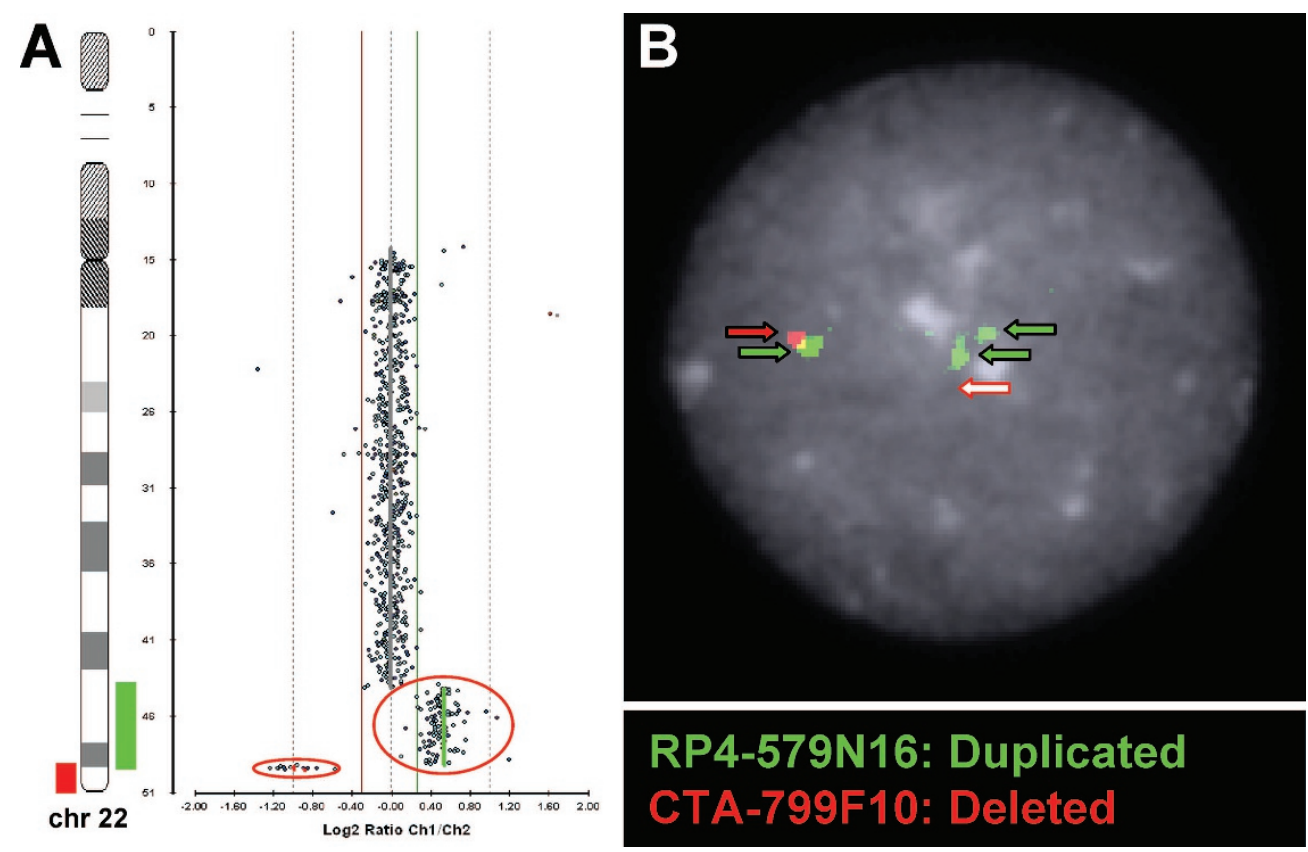

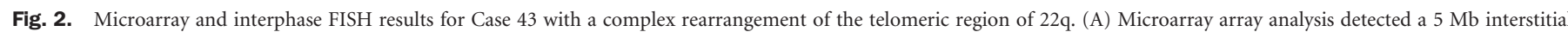

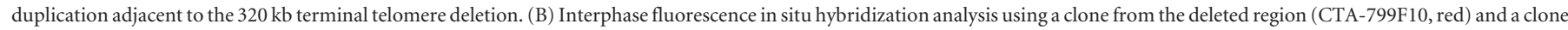

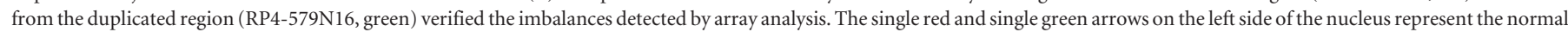

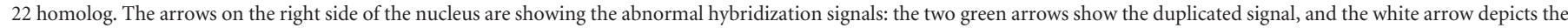
deleted signal.

Table 2

Benign telomere imbalances sized by FISH and oligonucleotide aCGH

\begin{tabular}{|c|c|c|c|c|}
\hline \multirow[b]{2}{*}{ Case no. } & \multirow[b]{2}{*}{ Telomere } & \multicolumn{2}{|c|}{ Size } & \multirow[b]{2}{*}{$\begin{array}{c}\text { Mapped } \\
\text { by }\end{array}$} \\
\hline & & $\begin{array}{l}\text { Deletion } \\
(\mathrm{Mb})\end{array}$ & $\begin{array}{c}\text { Duplication } \\
(\mathrm{Mb})\end{array}$ & \\
\hline \multicolumn{5}{|l|}{ Deletions } \\
\hline 45 & $4 \mathrm{q}$ & 1.0 & & aCGH \\
\hline 46 & $9 p$ & $300 \mathrm{~kb}$ & & $\mathrm{aCGH}$ \\
\hline 47 & $10 \mathrm{q}$ & 1.3 & & $\mathrm{aCGH}$ \\
\hline 48 & $10 q$ & $100 \mathrm{~kb}$ & & FISH \\
\hline 49 & $21 q$ & 1.6 & & aCGH \\
\hline 50 & $\mathrm{Yq}$ & $317 \mathrm{~kb}$ & & $\mathrm{aCGH}$ \\
\hline \multicolumn{5}{|c|}{ Duplications } \\
\hline 51 & $3 p$ & & 1.5 & $\mathrm{aCGH}$ \\
\hline 52 & $4 q$ & & 1.2 & $\mathrm{aCGH}$ \\
\hline 53 & $10 q$ & & $170 \mathrm{~kb}$ & $\mathrm{aCGH}$ \\
\hline
\end{tabular}

Derivative

$54 \quad 12 \mathrm{p} ; 15 \mathrm{q} \quad$ None $(12 \mathrm{p}) \quad 538 \mathrm{~kb}(15 \mathrm{q}) \quad$ aCGH

FISH, fluorescence in situ hybridization; aCGH, array comparative genomic hybridization.

deletion. In contrast, all the benign variants were $<2 \mathrm{Mb}$ in size. Figure 3 summarizes the size distribution of pathogenic and benign telomere imbalances and illustrates the size distinction between these two categories.

\section{DISCUSSION}

This study used molecular ruler analysis with FISH or array CGH analysis to characterize the size of telomere imbalance in 54 individuals with developmental delay/mental retardation who previously had normal G-banding analysis. The telomere imbalances examined included pure deletions and duplications as well as derivative chromosomes involving more than one rearrangement. Although these studies were initiated using FISH analysis as a primary method, it quickly became apparent that more efficient methods were necessary for high-resolution mapping of telomere imbalances.

Therefore, we transitioned our molecular ruler analysis for telomeres from FISH to array CGH by creating a custom oligonucleotide array with high-density coverage of each telomere region. FISH analysis is labor-intensive for fine mapping studies because multiple hybridizations are necessary for each case, which increases the analysis time and is subject to an individual's interpretation. In addition, duplications are hard to identify and delineate using FISH analysis. Unlike FISH analysis, array CGH provides an objective method for the efficient and accurate characterization of telomere imbalances in a single experiment. In addition, because of the ease of adding increased genome coverage with oligonucleotide-based arrays, additional imbalances can be accurately detected that could be missed with FISH or arrays containing lower resolution telomere coverage. However, as demonstrated by Case 45 (Fig. 1, D), careful evaluation of the telomeric content of microarrays is needed because pathogenic imbalances could be missed 


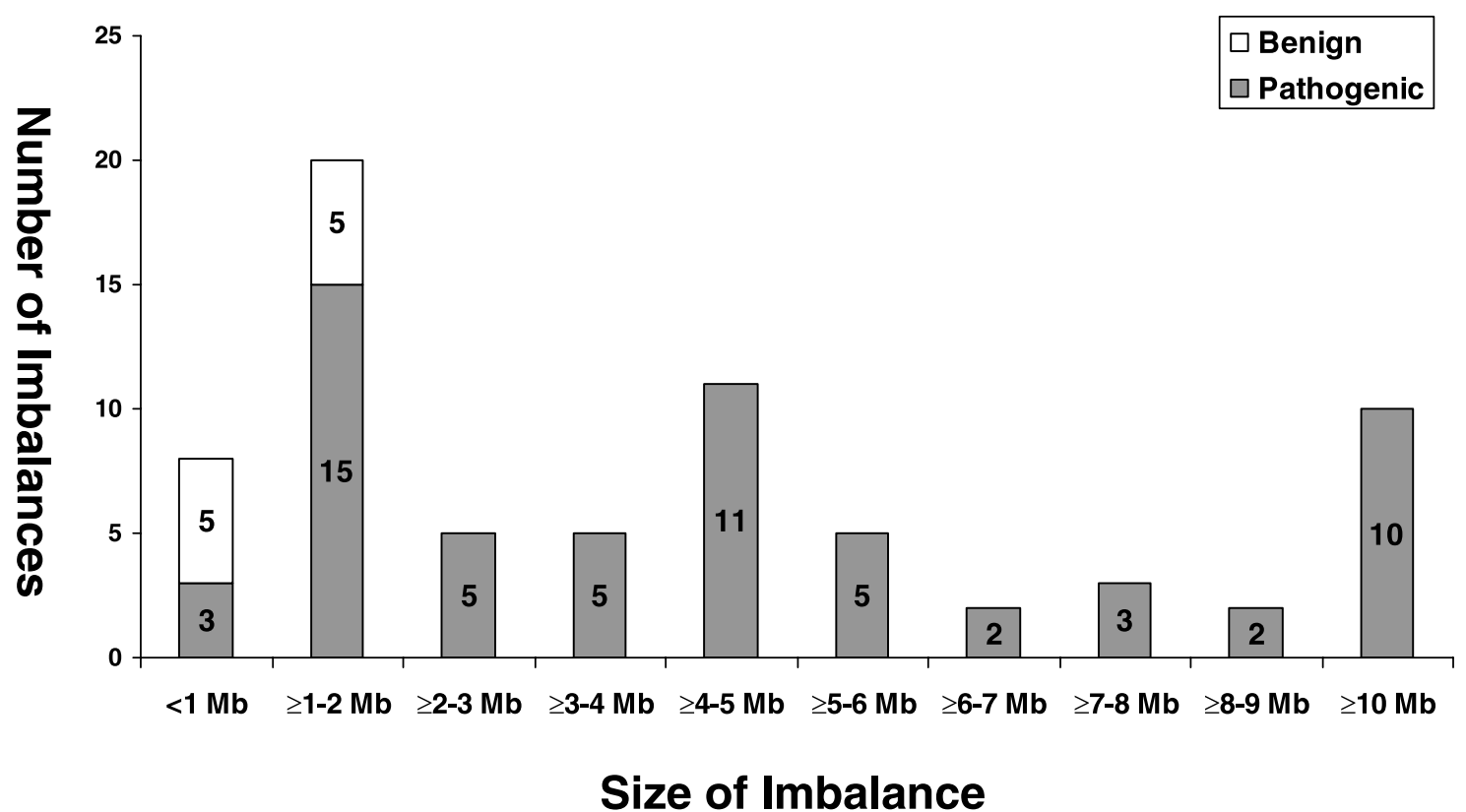

Fig. 3. The size distribution of pathogenic and benign telomere imbalances mapped by molecular ruler analysis. All the benign variants were $<2 \mathrm{Mb}$ in size. The pathogenic imbalances ranged from $<1 \mathrm{Mb}$ to $>10 \mathrm{Mb}$.

without adequate coverage that extends to the most distal unique DNA for each telomere region.

High-resolution mapping of telomere imbalances that are either causative of a clinical phenotype or represent benign variants is useful for developing gene dosage maps. Such detailed characterization of the genes that are sensitive to dosage changes (haploinsufficient or triplosensitive) versus those that are tolerant will be instrumental in the elucidation of genotype/phenotype correlations. As more individuals are studied with telomere imbalances involving each telomere region, additional information can be gained that will aid in providing better prognostic information related to each unique imbalance. For example, in this study, the size of pathogenic imbalances ranged from $400 \mathrm{~kb}$ to $13.5 \mathrm{Mb}$, whereas all the benign variants examined were $<1.6 \mathrm{Mb}$ in size. The smallest de novo deletion was $400 \mathrm{~kb}$ of the $6 \mathrm{q}$ telomere region in a male with developmental delay, short stature, and congenital anomalies. The deleted segment in this individual only includes three genes (PSMB1, PDCD2, and TBP); however, this small deletion results in a severe clinical phenotype. In contrast, a terminal deletion of $1.6 \mathrm{Mb}$ of $21 \mathrm{q}$ was shown to be an inherited benign variant that includes $\sim 15$ known genes.

In the current study, we identified and characterized 10 cases with benign familial variants that had deletions or duplications of the telomeric regions of chromosomes $3 \mathrm{p}, 4 \mathrm{q}, 9 \mathrm{p}$, $10 \mathrm{q}, 15 \mathrm{q}, 21 \mathrm{q}$, and Yq. Imbalances in the telomeric regions have previously been reported in normal subjects without any apparent phenotype. ${ }^{5,24-26}$ The absence of phenotypic effects is most likely related to the size and gene content of the genomic region involved in the imbalance. From our data and those of others, benign variants $>2 \mathrm{Mb}$ in size are very rare (Database of Genomic Variants, http://projects.tcag.ca/variation/). In addition, many genes in the genome are likely not dosage sensitive (e.g., genes for autosomal recessive conditions); therefore, copy number changes of these regions would be predicted to not cause an abnormal clinical phenotype. Additional detailed characterization of telomeric imbalances with and without phenotypic effects will aid in the definition of how much monosomy or trisomy can be tolerated without phenotypic effects.

We originally predicted that most cryptic telomere imbalances that were identified in individuals with developmental delay/mental retardation and a normal karyotype would be $<1$ $\mathrm{Mb}$ in size. ${ }^{6}$ However, our detailed mapping results presented here and in previous publications $s^{11,13}$ and those of Ballif et al. ${ }^{14}$ have demonstrated that imbalances of this size actually account for the minority of cases. In fact, $36 \%$ of our cases and $44 \%$ of those of Ballif et al. had imbalances that extended beyond $5 \mathrm{Mb}$. Thus, many telomere imbalances are quite large and still not detectable by G-banding analysis, which has been deemed the gold standard for detecting chromosomal gains or losses.

Furthermore, using high-definition telomere coverage with oligonucleotide arrays, we identified four cases with additional imbalances that were not detected using a single genomic clone for telomere analysis. Each of these cases had additional duplications of genomic material that were located adjacent to the deleted telomere region. This phenomenon has previously been reported by high-resolution characterization of specific cases involving terminal rearrangements, including deletions and ring chromosomes. ${ }^{27-30}$ Additional imbalances such as these are important to identify because they may modify the clinical presentation of an individual. Microarray analysis will 
facilitate such detailed studies to become part of routine clinical practice.

Given these results and those of Ballif et al., ${ }^{14}$ the sensitivity and reliability of the cytogenetic gold standard are being challenged by results from new molecular cytogenetic methods. Detailed characterization of telomere imbalances alone has demonstrated that the resolution of G-banding analysis is not as high as previously predicted and clearly the lower limit of G-banding detection is well above the threshold for significant phenotypic effects. This information for the telomere regions is likely reflective of imbalances across the whole genome. Therefore, higher resolution methods, such as microarray analysis, are necessary for a more sensitive methodology to detect clinically relevant chromosomal imbalances.

\section{ACKNOWLEDGMENTS}

This work was supported by NIH grant RO1 MH074090 (to C.L.M. and D.H.L.). The authors acknowledge JiYun Lee, $\mathrm{PhD}$, Brian Bunke, BS, and Joshua Lowman, BS, for expert technical assistance. They also thank the physicians and genetic counselors who referred patients and the families with telomere imbalances who participated in this study.

\section{References}

1. Ledbetter DH. Minireview: cryptic translocations and telomere integrity. Am J Hum Genet 1992;51:451-456.

2. Collaboration NIoHaIoMM. A complete set of human telomeric probes and their clinical application. National Institutes of Health and Institute of Molecular Medicine collaboration. Nat Genet 1996;14:86-89.

3. Knight SJ, Lese CM, Precht KS, Kuc J, et al. An optimized set of human telomere clones for studying telomere integrity and architecture. Am J Hum Genet 2000;67: 320-332.

4. Knight SJ, Regan R, Nicod A, Horsley SW, et al. Subtle chromosomal rearrangements in children with unexplained mental retardation. Lancet 1999;354: 1676-1681.

5. Ravnan JB, Tepperberg JH, Papenhausen P, Lamb AN, et al. Subtelomere FISH analysis of 11688 cases: an evaluation of the frequency and pattern of subtelomere rearrangements in individuals with developmental disabilities. J Med Genet 2006;43: 478-489.

6. Veltman JA, Schoenmakers EF, Eussen BH, Janssen I, et al. High-throughput analysis of subtelomeric chromosome rearrangements by use of array-based comparative genomic hybridization. Am J Hum Genet 2002;70:1269-1276.

7. Harada N, Hatchwell E, Okamoto N, Tsukahara M, et al. Subtelomere specific microarray based comparative genomic hybridisation: a rapid detection system for cryptic rearrangements in idiopathic mental retardation. J Med Genet 2004;41:130136.

8. Wong A, Lese Martin C, Heretis K, Ruffalo T, et al. Detection and calibration of microdeletions and microduplications by array-based comparative genomic hy- bridization and its applicability to clinical genetic testing. Genet Med 2005;7:264271.

9. Cheung SW, Shaw CA, Yu W, Li J, et al. Development and validation of a CGH microarray for clinical cytogenetic diagnosis. Genet Med 2005;7:422-432.

10. Shaffer LG, Kashork CD, Saleki R, Rorem R, et al. Targeted genomic microarray analysis for identification of chromosome abnormalities in 1500 consecutive clinical cases. J Pediatr 2006;149:98-102.

11. Martin CL, Waggoner DJ, Wong A, Uhrig S, et al. "Molecular rulers" for calibrating phenotypic effects of telomere imbalance. J Med Genet 2002;39:734-740.

12. Cardoso C, Leventer RJ, Ward HL, Toyo-Oka K, et al. Refinement of a 400-kb critical region allows genotypic differentiation between isolated lissencephaly, Miller-Dieker syndrome, and other phenotypes secondary to deletions of $17 \mathrm{p} 13.3$. Am J Hum Genet 2003;72:918-930.

13. Eash D, Waggoner D, Chung J, Stevenson D, et al. Calibration of $6 \mathrm{q}$ subtelomere deletions to define genotype/phenotype correlations. Clin Genet 2005;67:396-403.

14. Ballif BC, Sulpizio SG, Lloyd RM, Minier SL, et al. The clinical utility of enhanced subtelomeric coverage in array CGH. Am J Med Genet 2007;143:1850-1857.

15. Toruner GA, Streck DL, Schwalb MN, Dermody JJ. An oligonucleotide based arrayCGH system for detection of genome wide copy number changes including subtelomeric regions for genetic evaluation of mental retardation. Am J Med Genet A 2007;143:824-829.

16. Kent WJ, Sugnet CW, Furey TS, Roskin KM, et al. The human genome browser at UCSC. Genome Res 2002;12:996-1006.

17. Karolchik D, Hinrichs AS, Furey TS, Roskin KM, et al. The UCSC Table Browser data retrieval tool. Nucleic Acids Res. 2004;32:D493-D496.

18. Lander ES, Linton LM, Birren B, Nusbaum C, et al. Initial sequencing and analysis of the human genome. Nature 2001;409:860-921.

19. Archer HL, Gupta S, Enoch S, Thompson P, et al. Distinct phenotype associated with a cryptic subtelomeric deletion of 19p13.3-pter. Am J Med Genet A 2005;136:38-44.

20. Sebat J, Lakshmi B, Troge J, Alexander J, et al. Large-scale copy number polymorphism in the human genome. Science 2004;305:525-528.

21. Iafrate AJ, Feuk L, Rivera MN, Listewnik ML, et al. Detection of large-scale variation in the human genome. Nat Genet 2004;36:949-951.

22. Tuzun E, Sharp AJ, Bailey JA, Kaul R, et al. Fine-scale structural variation of the human genome. Nat Genet 2005;37:727-732.

23. Redon R, Ishikawa S, Fitch KR, Feuk L, et al. Global variation in copy number in the human genome. Nature 2006;444:444-454.

24. Ballif BC, Kashork CD, Shaffer LG. The promise and pitfalls of telomere regionspecific probes. Am J Hum Genet 2000;67:1356-1359.

25. Knight SJ, Flint J. Perfect endings: a review of subtelomeric probes and their use in clinical diagnosis. J Med Genet 2000;37:401-409.

26. Joyce CA, Dennis NR, Cooper S, Browne CE. Subtelomeric rearrangements: result from a study of selected and unselected probands with idiopathic mental retardation and control individuals by using high-resolution G-banding and FISH. Hum Genet 2001;109:440-451.

27. Ballif BC, Yu W, Shaw CA, Kashork CD, et al. Monosomy 1 p36 breakpoint junctions suggest pre-meiotic breakage-fusion-bridge cycles are involved in generating terminal deletions. Hum Mol Genet 2003;12:2153-2165.

28. Yu W, Ballif BC, Kashork CD, Heilstedt HA, et al. Development of a comparative genomic hybridization microarray and demonstration of its utility with 25 wellcharacterized 1p36 deletions. Hum Mol Genet 2003;12:2145-2152.

29. Jobanputra V, Sebat J, Troge J, Chung W, et al. Application of ROMA (representational oligonucleotide microarray analysis) to patients with cytogenetic rearrangements. Genet Med 2005; 7:111-118.

30. Knijnenburg J, van Haeringen A, Hansson KB, Lankester A, et al. Ring chromosome formation as a novel escape mechanism in patients with inverted duplication and terminal deletion. Eur J Hum Genet 2007;15:548-555. 